\title{
A Novel High-Sensitivity Rapid-Acquisition Single-Photon Cardiac Imaging Camera
}

Sanjiv S. Gambhir ${ }^{1}$, Daniel S. Berman ${ }^{2}$, Jack Ziffer ${ }^{3}$, Michael Nagler ${ }^{4}$, Martin Sandler ${ }^{5}$, Jim Patton ${ }^{5}$, Brian Hutton ${ }^{6}$, Tali Sharir ${ }^{7}$, Shlomo Ben Haim ${ }^{4}$, and Simona Ben Haim ${ }^{6}$

${ }^{I}$ Departments of Radiology and Bioengineering, Molecular Imaging Program, Stanford University, Stanford, California; ${ }^{2}$ Department of Imaging and Department of Medicine (Division of Cardiology), Cedars-Sinai Medical Center, Los Angeles, California; ${ }^{3}$ Baptist Hospital of Miami, Miami, Florida; ${ }^{4}$ Spectrum Dynamics, Ltd., Tirat Hacarmel, Israel, ${ }^{5}$ Department of Radiology and Radiological Sciences, Vanderbilt University Medical Center, Nashville, Tennessee; ${ }^{6}$ Institute of Nuclear Medicine, University College London and University College London Hospitals NHS Trust, London, United Kingdom; and

${ }^{7}$ Procardia-Maccabi Healthcare Services, Tel Aviv, Israel

This study described and validated a new solid-state singlephoton $\gamma$-camera and compared it with a conventional-SPECT Anger camera. The compact new camera uses a unique method for localizing $\gamma$-photon information with a bank of 9 solid-state detector columns with tungsten collimators that rotate independently. Methods: Several phantom studies were performed comparing the new technology with conventional-SPECT technology. These included measurements of line sources and single- and dual-radionuclide studies of a torso phantom. Simulations were also performed using a cardiothoracic phantom. Furthermore, 18 patients were scanned with both the new camera and a conventional-SPECT camera. Results: The new camera had a count sensitivity that was 10 times higher than that of the conventional camera and a compensated spatial resolution that was moderately better. Dual-radionuclide studies using a phantom show the further potential of the new camera for a 2-tracer simultaneous acquisition. Two-minute clinical studies with the new camera and 11-min studies with the conventional camera qualitatively showed good-to-excellent image quality and improved myocardial edge definition for the new camera. Conclusion: These initial performance characteristics of a new solid-state single-photon $\gamma$-camera offer great promise for clinical dynamic SPECT protocols, with important implications for applications in nuclear cardiology and molecular imaging.

Key Words: molecular imaging; myocardial perfusion SPECT; nuclear cardiology

J Nucl Med 2009; 50:635-643

DOI: 10.2967/jnumed.108.060020

$\mathbf{N}$ discipline for the noninvasive assessment of physiologic and molecular function in normal and diseased tissues. Principally performed with nanomolar quantities of administered

Received May 28, 2008; revision accepted Dec. 29, 2008.

For correspondence or reprints contact: Sanjiv Sam Gambhir, James H. Clark Center, Departments of Radiology and Bioengineering, Stanford University School of Medicine, 318 Campus Dr., Clark E150, Stanford,

CA 94305-5427.

E-mail: sgambhir@stanford.edu

COPYRIGHT () 2009 by the Society of Nuclear Medicine, Inc. radiopharmaceuticals and an external scintillation camera, nuclear medicine imaging uses 2 types of modalities: singlephoton imaging (including planar imaging and SPECT) and PET, with the former comprising nearly three fourths of all clinical procedures. With SPECT, myocardial perfusion studies predominate; these studies were performed in approximately 7,000,000 patients in the United States in 2004 and provided images of relative myocardial perfusion at rest and under stress. By assessing the extent of ischemic and infarcted myocardium, SPECT provides noninvasive information that has become central in clinical decision making, determining the need for invasive cardiac catheterization and myocardial revascularization in many patients $(1,2)$.

SPECT is typically performed using an Anger scintillation camera, named after its inventor, Hal Anger (3). Most myocardial SPECT is performed with 2 scintillation cameras oriented at $90^{\circ}$ and mounted on a gantry that rotates around the patient. Typically, each scintillation camera is equipped with parallel-hole high-resolution collimators. Since collimation is necessary to acquire the projection views, only $0.02 \%$ of the photons emitted from the heart are collected. As a result, acquisition times of 10-20 min are required for myocardial SPECT studies. Although new detector technologies using solid-state materials have been explored (4-6) and have some clinical applications (7-9), they have not significantly improved system sensitivity, which is limited primarily by collimation (10-13).

A novel photon-collection and scanning geometry that includes position and orientation tracking of the detectors and unique reconstruction algorithms has been developed in the current work to improve the built-in trade-off between spatial resolution and sensitivity. In addition, this new development applies resolution recovery in a way that allows high-sensitivity collimators to be used for radionuclide imaging while maintaining excellent spatial resolution. Similar approaches have been developed by other investigators in the context of small-animal SPECT (14) and now are being used in clinical SPECT (15). A marked increase in sensitivity 
is afforded by this approach, enabling the SPECT time or administered tracer dose to be greatly reduced or offering the potential for rapid acquisition of dynamic SPECT data after radiotracer injection. The dynamic SPECT afforded by this new approach has resulted in the term D-SPECT being applied to the camera (Spectrum Dynamics). This paper initially describes and validates the D-SPECT camera system, using simulation, various physical phantoms, and pilot patient data that compare the performance of the D-SPECT camera with conventional SPECT cameras.

\section{MATERIALS AND METHODS}

\section{The D-SPECT Camera}

The novel D-SPECT camera technology uses 9 collimated detector columns arranged in a curved configuration to conform to the shape of the left side of the patient's chest (Fig. 1). The tungsten parallel-hole collimators used by Spectrum Dynamics are shorter $(21.7 \mathrm{~mm})$ and have larger square holes $(2.26 \mathrm{~mm})$ than do the standard lead parallel-hole collimators (45-mm length and 1.6-mm holes for a low-energy high-resolution [LEHR] collimator) used for conventional SPECT. The result is an acceptance solid angle $\left(10.847 \times 10^{-3} \mathrm{sr}\right)$ more than 8 times that of the standard LEHR lead parallel-hole SPECT collimator $\left(1.264 \times 10^{-3} \mathrm{sr}\right)$. An array of cadmium zinc telluride (CZT) crystals is aligned behind each collimator column (width, 16 pixels [40 mm]; length, 64 pixels [160 mm]). The square collimator holes are in registration with the crystal detector array, with $1 \mathrm{CZT}$ pixel for each hole (Fig. 1). Each collimated detector column rotates and translates (a maximum of $110^{\circ}$ ) independently, either stepwise or continuously, allowing the object of interest to be viewed from hundreds of different viewing angles (Supplemental Video 1; supplemental materials are available online only at http://jnm.snmjournals.org). This sampling strategy leads to a complete dataset as determined by a sinogram (Supplemental Fig. 1). As currently configured, the columns can go through their full rotation, enabling dynamic SPECT acquisitions with a 10-s temporal resolution. A time marker is input every $10 \mathrm{~ms}$, and the data are acquired in list mode using a standard personal computer (Pentium IV; Intel) (3-GHz clock rate, 2-GB memory). Physiologic markers, such as the electrocardiogram $\mathrm{R}$ wave, can also be routinely recorded with the system.

\section{Image Reconstruction}

The acquired data for the D-SPECT camera are reconstructed using an iterative algorithm based on the maximum-likelihood expectation maximization (MLEM) method. The probability of a $\gamma$-ray's having been emitted from coordinates $(x, y, z)$ relative to the detector is modeled, creating a probability map used by the reconstruction algorithm. The characteristics of the collimator are included in the probability map estimation (16-20), but attenuation and scatter are not included.

Although MLEM iteration is guaranteed to improve the likelihood of the estimate, convergence to a maximum-likelihood solution may be slow. To accelerate the convergence of the MLEM algorithm, we have applied an ordered-subset expectation maximization (OSEM) (20) approach. The subsets are chosen on the basis of the structure of the D-SPECT camera and on the scanning scheme, so that each voxel has a similar influence on each of the subsets. The order of the computation is chosen so as to minimize the dependency between successive readings.

Likelihood maximization is combined with a technique that has been developed specifically for the cardiac application and that globally smoothes intensity levels over the voxels corresponding to the left ventricular (LV) walls at an intermediate stage of the reconstruction. These voxels are located in the reconstructed volume after several OSEM iterations by searching radially from the geometric center of the LV cavity, taking the points of maximal intensity, and fitting a smooth ellipsoid-like surface to these points. Further iterations are then performed. During reconstruction, spatial smoothing is applied every $n$ iterations to the current partial result. Smoothing is performed by replacing the value of each voxel value $\mathrm{V}_{0}$ by a value $\mathrm{V}_{1}$ that is computed as follows: $\mathrm{V}_{1}=\mathrm{w} \times \mathrm{N}+(1-\mathrm{w})$ $\times V_{0}$, where $N$ is the 26-neighborhood average of the voxel and $w$ is the weight between 0 and 1 . A mild post-reconstruction filter is also applied to suppress hot spots by detecting and flattening local maxima.

Spatial resolution effects due to the collimation are referred to as geometric or extrinsic. The intrinsic resolution is determined by the CZT detector size. Accurate modeling of the probability function, supported by an increased number of viewing angles, overcomes the loss of geometric spatial resolution inherent with wider-bore collimation and recovers resolution. For a standard SPECT camera with one or more large planar detectors positioned at a given angle, all the pixels at a given head position collect photons during the same time

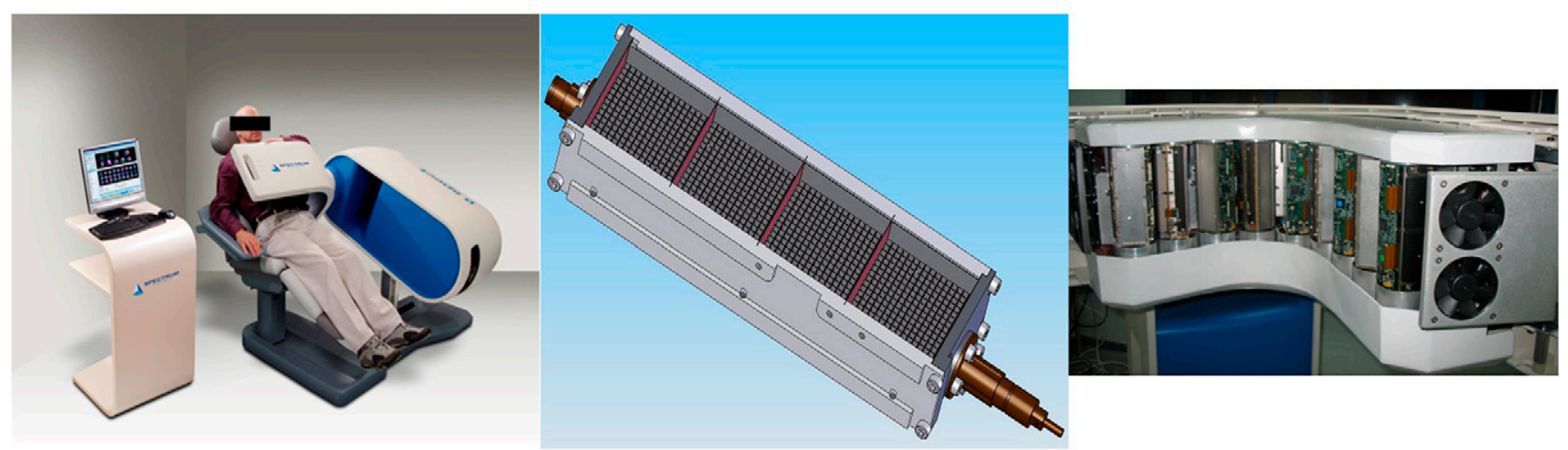

FIGURE 1. D-SPECT cardiac system and detector-head configuration. (Left) D-SPECT cardiac scanner with seated patient. (Middle) Each detector column is composed of CZT sensor $(39 \times 39 \times 5 \mathrm{~mm})$ with four $16 \times 16$ pixel detectors and tungsten collimator with 0.2-mm septa and square opening (pitch, $2.46 \mathrm{~mm}$; length, $21.7 \mathrm{~mm}$ ). (Right) Nine detector columns, each capable of rotation and translation, are used to scan the myocardium. 
interval. The same collection time is given to pixels that denote informative regions (such as at the left ventricle) as is given to pixels that denote less significant regions (such as a body contour, at the edge of the detector). The design of the D-SPECT camera mechanics allows us to overcome this inherent SPECT limitation. By choosing to spend more time directing the independent small detector heads of the D-SPECT camera toward regions of interest, one can allocate more time to collecting data from these regions at the expense of collecting fewer data from less important regions. This design is equivalent to having a single detector head, with each of its pixels having a different time for data collection.

To help the reader better understand this idea, we introduce the terms equivalent projection head and virtual detector head. An equivalent projection is a 2-dimensional image generated by averaging onto a single plane the projection images from multiple D-SPECT detector heads sharing the same viewing angle. This plane can be referred to as a virtual single-head detector. Because D-SPECT detectors may have different dwell times for the same viewing angle, each pixel value on this virtual detector is obtained by averaging a different number of projections. The equivalent projection (Fig. 2A) is obtained by dividing the sum of D-SPECT projections (Fig. 2B) by the dwell times (Fig. 2C). Figure 2C shows that pixels looking at the left ventricle average $4-8$ projections, pixels looking near the left ventricle average $2-3$ projections, and pixels far from the left ventricle are not observed (Fig. 2C). The D-SPECT algorithm has been further refined for optimized reconstruction of clinical data by incorporating a prior specifically for this application.

\section{Acquisition Protocol}

National Electrical Manufacturers Association (NEMA) phantom and patient feasibility studies were performed, comparing the high-speed D-SPECT camera technology with that of a typical dualdetector Anger camera (Millennium VG dual-head $\gamma$-camera; GE Healthcare) equipped with a LEHR (model VPC-45; GE Healthcare) collimator. The usable field of view (UFOV) was mapped for the D-SPECT camera. For the conventional-SPECT phantom studies, the dual-head Anger camera was positioned in the L-mode with a $100-\mathrm{mm}$ radius of rotation; a $180^{\circ}$ scan was obtained, with an angular step of $3^{\circ}$, mimicking clinical cardiac SPECT acquisition. For the D-SPECT phantom studies, each detector was rotated through an arc ranging from $60^{\circ}$ to $110^{\circ}$, depending on the position of the individual detector and the scanned object. The energy window for each camera was centered around $122 \mathrm{keV} \pm 10 \%$ for the ${ }^{57} \mathrm{Co}$ for phantom studies, $140 \mathrm{keV} \pm 10 \%$ for the ${ }^{99 \mathrm{~m}} \mathrm{Tc}$ patient studies, and $70 \mathrm{keV} \pm 15 \%$ for the ${ }^{201} \mathrm{Tl}$ portion of the dualradionuclide studies.

\section{Line-Source Studies}

A $1.48 \times 10^{8} \mathrm{~Bq}{ }^{57} \mathrm{Co}$ line source (diameter, $1 \mathrm{~mm}$; length, 190 $\mathrm{mm}$ ) demonstrated the inherent spatial resolution and sensitivities of the 2 systems. With each system, $14.3 \times 10^{3}$ counts were obtained. The time of acquisition was measured, and the resolution was measured as the full width at half maximum (FWHM) and full width at tenth maximum (FWTM).

A standard NEMA phantom with three ${ }^{99 \mathrm{~m}} \mathrm{Tc}$ line sources (each source: diameter, $1 \mathrm{~mm}$; length, $20 \mathrm{~cm}$ ) demonstrated the extrinsic spatial resolution of the 2 systems. For both systems-D-SPECT and SPECT - scans were obtained under conditions optimal for cardiac acquisition (Supplemental Fig. 2). On the basis of the collimator design, the expected geometric resolution (FWHM) for the D-SPECT collimator is more than a factor of 2 worse than that of the LEHR collimator.

Standard NEMA protocols define a reconstruction method based on filtered backprojection, with a ramp filter that is clearly inappropriate for the D-SPECT camera (for which an iterative reconstruction algorithm is intrinsic to the system). Therefore, conventional-SPECT data were reconstructed with 20-iteration OSEM using $1.73-\mathrm{mm}$ cubic voxels (matrix size, $256 \times 256$ ) with no resolution recovery. The D-SPECT data reconstruction also used 20 iterations of OSEM, but with 2.46-mm cubic voxels and with resolution recovery obtained by the inclusion of collimator characteristics in the imaging model. With each system, time of acquisition and spatial resolution (FWHM, FWTM) were measured.

The mapping of the resolution throughout a UFOV of the D-SPECT camera was performed by imaging a ${ }^{57} \mathrm{Co} 9$-rod grid placed in the

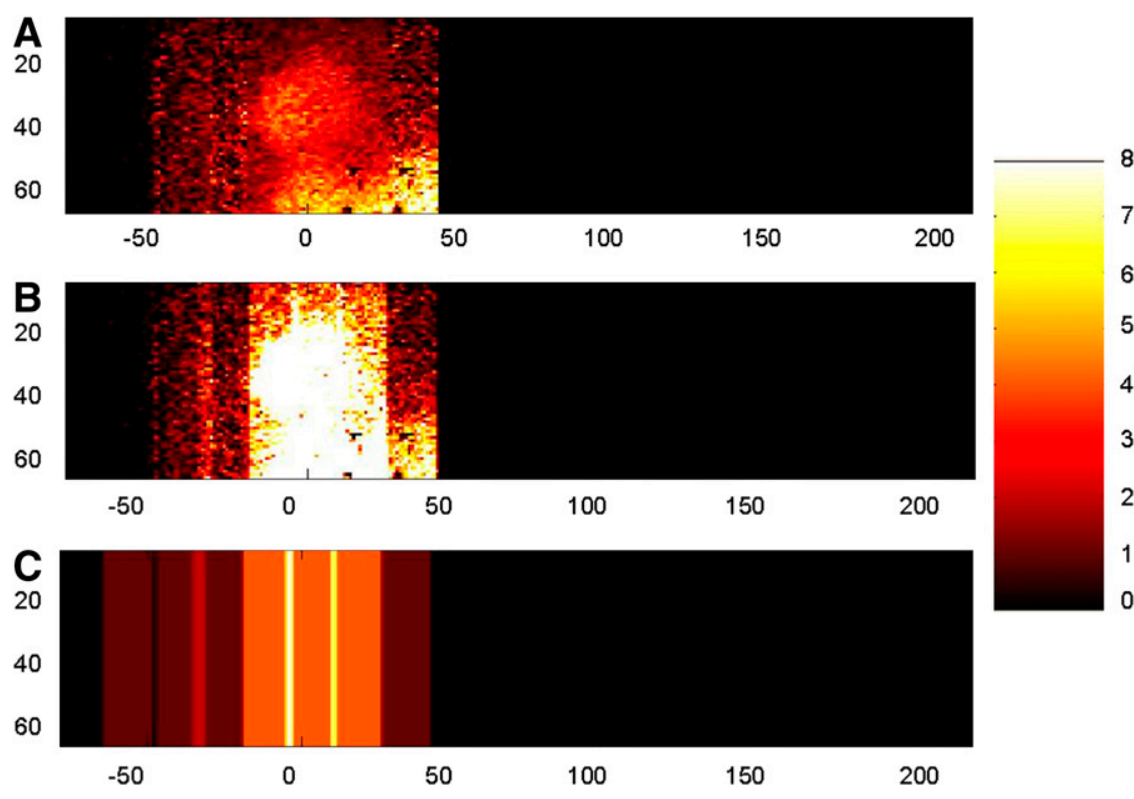

FIGURE 2. A 2-dimensional image generated by averaging projection images from multiple D-SPECT detector heads, sharing same viewing angle, onto single plane from virtual singlehead detector. Equivalent projection $(A)$ is obtained by dividing sum of D-SPECT projections $(\mathrm{B})$ by dwell times $(\mathrm{C})$. 
UFOV (Supplemental Fig. 3). Each ${ }^{57}$ Co rod was $1 \mathrm{~mm}$ in diameter, with an activity of $0.74 \times 10^{8} \mathrm{~Bq}$. The acquisition protocol entailed 60 positions and $2 \mathrm{~s}$ per position. The total acquisition time was $120 \mathrm{~s}$. The experiment was performed for the D-SPECT camera only, to demonstrate the stability and degradation of the resolution as a function of distance from the detectors.

\section{Reconstructed Spatial Resolution Estimation}

Resolution measurement using iterative reconstruction for linesource data tends to provide a poor estimate of the effective resolution in clinical practice; thus, we also used an alternative approach based on perturbation. A small change in the image distribution (i.e., the perturbation) provides a measure of the resolution determined by the underlying activity distribution, without significantly affecting the result.

A simplified model of the left ventricle, located in a thoracic cavity similar to that used in the NCAT (NURBS-based cardiac thoracic) phantom, was simulated $(21,22)$. This model was chosen as it resembled a physical phantom that could be used to perform similar estimation. Radioactivity was introduced to the ventricular wall, liver, and background regions (excluding lung), with relative concentrations of 6.5:2.5:0.2. Data were projected to simulate the acquisition geometry for both SPECT and D-SPECT cameras. In addition, a pair of radioactive wires, located in the center of the ventricular wall and extending around the short axis and long axis of the ventricle, was simulated (Fig. 3).

The activity concentration in the wires was scaled to $10 \%$ of the activity concentration in the ventricular wall, and a second set of projections was produced incorporating the active wires. Attenuation and scatter were not modeled. Supplemental Figure 2 illustrates the acquisition geometry. Both datasets were reconstructed using OSEM. For SPECT, the reconstruction was performed using 15 subsets, both with and without collimator modeling, using 2 and 54 iterations, respectively. For the D-SPECT camera, the collimator was modeled and reconstruction involved 32 subsets with 20 iterations. In the conventional-SPECT system, the geometric resolution was modeled as a gaussian function whose width varies linearly with the distance from the detector. The system resolution is then based on the geometric and intrinsic components added in quadrature (11). To evaluate resolution, attenuation and scatter were not modeled. The same assumptions were made for the D-SPECT camera simulations. In each case, the reconstructions with and without the active wire were subtracted to provide images that could yield measurements of line width. These measurements were obtained by first reorienting the heart so that
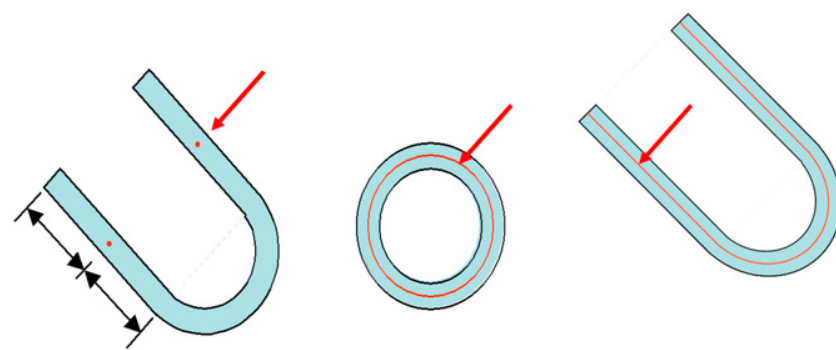

FIGURE 3. Simulated resolution estimation model. Simplified model of left ventricle with active wires was simulated (red arrow), located in the center of the ventricular wall and extending around the short and long axes of the ventricle. the long axis was vertically aligned; FWHM and FWTM were estimated radially (across the width of the ventricular wall) and tangentially (orthogonal to radial) and averaged for the complete wire length.

\section{Torso Phantom Studies}

An anthropomorphic torso phantom (model ECT/TOR/P; Data Spectrum Corp.) was used for the phantom studies (Supplemental Fig. 4). The phantom, a large, body-shaped cylinder, comprised the lungs (filled with Styrofoam beads [The Dow Chemical Co.]), liver, spine inserts, and cardiac inserts (model ECT/CAR/I), simulating the upper torso of average-to-large patients $(38 \times 26 \mathrm{~cm})$. The torso phantom was filled with ${ }^{99 \mathrm{~m}} \mathrm{Tc}$ with the following activity distributions: cardiac insert, $185 \mathrm{kBq} / \mathrm{mL}(5 \mu \mathrm{Ci} / \mathrm{mL})$; liver, $92.5 \mathrm{kBq} / \mathrm{mL}$ $(2.5 \mu \mathrm{Ci} / \mathrm{mL})$; and background and left ventricle cavity, $7.4 \mathrm{kBq} / \mathrm{mL}$ $(0.2 \mu \mathrm{Ci} / \mathrm{mL})$, resulting in the following signal-to-background ratios: heart and background (body), 25; heart and liver, 2; and liver and background, 12.5. Cardiac phantom studies were performed with homogeneous activity in the left ventricle and the following defect sizes, activities, and locations: $0.5-\mathrm{cm}$ defect, $0 \%$ activity, anterior wall; $1.0-\mathrm{cm}$ defect, $0 \%$ activity, anterior and inferior walls; and $2.0-\mathrm{cm}$ defect, $50 \%$ activity, inferior wall.

In a separate experiment, the same torso phantom, with a $2-\mathrm{cm}$ $(100 \%)$ defect in the anterior wall, was filled with ${ }^{99 \mathrm{~m}} \mathrm{Tc}$ and ${ }^{201} \mathrm{Tl}$ with the following activity distributions: for single-isotope thallium imaging: left ventricle, 5.0 MBq of ${ }^{201} \mathrm{Tl}$; liver, $18.9 \mathrm{MBq}$ of ${ }^{201} \mathrm{Tl}$; and background, $37 \mathrm{MBq}$ of ${ }^{201} \mathrm{Tl}$; and for the simultaneous dualradionuclide protocol ( ${ }^{99 \mathrm{~m}} \mathrm{Tc}$ was added to ${ }^{201} \mathrm{Tl}$ ): left ventricle, 5.9 $\mathrm{MBq}$ of ${ }^{99 \mathrm{~m}} \mathrm{Tc}$; liver, $37 \mathrm{MBq}$ of ${ }^{99 \mathrm{~m}} \mathrm{Tc}$; and background, $23.7 \mathrm{MBq}$ of ${ }^{99 \mathrm{~m}} \mathrm{Tc}$. The acquisition protocol entailed 120 positions and $3 \mathrm{~s}$ per position. The total acquisition time was $360 \mathrm{~s}$.

\section{Patient Studies}

Ethics committee approval for the clinical investigation was obtained from the Procardia Center in Tel Aviv before volunteers were imaged. A total of 18 patients (15 men, 13 with known coronary artery disease) underwent one-day ${ }^{99 \mathrm{~m}} \mathrm{Tc}$-sestamibi stress/ rest SPECT. D-SPECT images were obtained within $30 \mathrm{~min}$ after SPECT. Stress and rest acquisition times were 19 and $11 \mathrm{~min}$, respectively, for SPECT and 4 and $2 \mathrm{~min}$, respectively, for D-SPECT. Images were visually analyzed using the 20-segment model to calculate summed stress scores (SSS) and summed rest scores (SRS) and an image quality scale of $1-5$ (1, poor; 5 , excellent); images were assessed for confidence of interpretation by 4 masked nuclear medicine physicians. The images were also read by 3 independent nuclear medicine physicians with no affiliations to Spectrum Dynamics. Myocardial counts per minute were calculated for both SPECT and D-SPECT. Imaging on the D-SPECT camera was performed with the patients sitting upright, with their arms resting in a comfortable position; imaging on the conventional-SPECT camera was performed with the patients supine, with their arms over their head. Conventional-SPECT images were acquired using a $180^{\circ}$ elliptical orbit. D-SPECT images were acquired in list mode along with $\mathrm{R}$ wave markers, with each detector acquiring images from 120 angles. No attenuation correction was used. SPECT images were reconstructed using an iterative algorithm, and D-SPECT images were reconstructed using the MLEM algorithm as described above. Reconstructed images from both systems were reoriented into standard cardiac short and long axis views for visual comparison. Counts per minute in the myocardium for both D-SPECT and SPECT acquisitions were determined by analysis of raw data images 
on the Xeleris workstation (version, 1.1324; computer model, XW6200; GE Healthcare). Eight-frame gating was applied to the SPECT images. Eight-frame gated images were reconstructed from the list-mode D-SPECT data and were compared visually with the gated SPECT data for image quality. LV ejection fraction and LV volumes were computed from the gated images of both systems using commercially available software (Quantitative Gated SPECT; Cedars-Sinai Health System) (22). LV ejection fractions and LV volumes from 2 patients with mild and severe perfusion defects were computed and compared.

\section{RESULTS}

\section{Line-Source Studies}

Using the single line source imaged with the D-SPECT camera, we obtained $14.3 \times 10^{6}$ events in $4 \mathrm{~min}$; with the conventional-SPECT camera, this acquisition took 45.5 min. The FWHM and FWTM of the single line source with D-SPECT were 3.5 and $6.8 \mathrm{~mm}$ in the $x$-axis and 4.2 and $7.9 \mathrm{~mm}$ in the $y$-axis, respectively; with SPECT, the FWHM and FWTM of the single line source were 9.2 and $16.6 \mathrm{~mm}$ in the $x$-axis and 12.5 and $22.3 \mathrm{~mm}$ in the $y$-axis, respectively (Supplemental Fig. 5).

Supplemental Figure 5 shows the reconstructed SPECT and D-SPECT images from the NEMA 3-line-source studies. The total acquisition time was $30 \mathrm{~min}$ for SPECT and $20 \mathrm{~min}$ for D-SPECT. The average FWHM and FWTM of the 3 line sources with D-SPECT were 3.9 and $7.5 \mathrm{~mm}$, and with SPECT they were 10.1 and 19.3 $\mathrm{mm}$, respectively (Supplemental Table 1). These measurements suggest a gain in D-SPECT spatial resolution, compared with that of SPECT, on the order of approximately 2.5. However, reconstruction of the SPECT data with collimator modeling was not performed and would have helped to improve the SPECT spatial resolution. A spatial resolution as good as that obtained with the D-SPECT camera using NEMA standards for Astonish processing. The normal radius of rotation for a $180^{\circ}$ orbit with optimal patient set-up is on the order of $20 \mathrm{~cm}$; at this radius, the SPECT reconstructed resolution using NEMA methods is about $12 \mathrm{~mm}$ for a high-resolution collimator. The D-SPECT camera does get closer than does the conventionalSPECT camera but also has a poorer geometric resolution. These measurements demonstrate the effectiveness of the DSPECT reconstruction algorithms in achieving spatial resolution recovery, but these values do not necessarily represent the spatial resolution obtained in clinical practice.

\section{UFOV Studies}

Mapping of the spatial resolution throughout a UFOV demonstrated an average spatial resolution (FWHM) of $3.93 \pm 0.82 \mathrm{~mm}$ and $4.59 \pm 0.74 \mathrm{~mm}$ in the $x$ - and $y$-axes, respectively. The FWTM is $8.01 \pm 1.43 \mathrm{~mm}$ and $9.21 \pm$ $1.22 \mathrm{~mm}$ in the $x$ - and $y$-axes, respectively (Supplemental Table 3).

\section{Reconstructed Spatial Resolution Estimation}

We next used a perturbation method to more realistically measure gains in the spatial resolution of the D-SPECT camera over the conventional-SPECT camera. Similar methods have been described by Stayman and Fessler (23). Detailed results from the perturbation experiments are presented in Supplemental Table 2. The reconstructed resolution for SPECT demonstrated a resolution for the long-axis wire (FWHM averaged for complete wire \pm SD) of $12.06 \mathrm{~mm}( \pm 0.42)$ radially and $11.37 \mathrm{~mm}( \pm 0.91)$ tangentially, without resolution modeling; the inclusion of the resolution model improved radial resolution to $7.80 \mathrm{~mm}$ ( \pm 0.32 ) but did not affect the tangential resolution (11.57 $\mathrm{mm}[ \pm 0.85])$. This result is perhaps not surprising, given that there was no structure inside the ventricular wall, and provides some level of confidence that measurements do reflect the underlying activity distribution. In the case of the D-SPECT for the same short-axis wire, radial resolution was $8.76 \mathrm{~mm}( \pm 1.65)$ and tangential resolution was 12.94 $\mathrm{mm}( \pm 2.0)$. Results for the short-axis wire were similar, as summarized in Supplemental Table 2, with slightly poorer tangential resolution overall. These results suggest that in practice the radial resolution of the D-SPECT camera is superior to that of the SPECT camera and approaches that obtained when resolution is modeled for SPECT with a high-resolution collimator. Once again, the tangential resolution is worse, although this measurement probably has little significance.

These results reconfirm that D-SPECT image reconstruction has spatial resolution superior to that obtained in conventional cardiac SPECT reconstruction, despite the poor geometric resolution for the D-SPECT collimator. The perturbation figures provide a better impression of the comparative resolution of the 2 systems in clinical practice, in which the reconstruction involves fewer iterations for practical reasons but also has a more complex activity distribution.

\section{Torso Phantom Studies}

Figure 4 shows images from the SPECT and D-SPECT scans of the torso phantom (Supplemental Fig. 4 also shows a photograph of the phantom) under several activity concentrations in the heart and liver, with and without myocardial defects. Supplemental Figure 6 shows additional examples of scans of the torso phantom. In each case, qualitative interpretation demonstrates the sharper edge definition of the D-SPECT images over the SPECT images because of the improved spatial resolution of the new technology. No problems in defect identification were encountered for either the SPECT or the D-SPECT images.

The CZT-based detectors used in the D-SPECT provide better energy resolution and reduce the isotope cross-talk phenomenon. Single-isotope $\left({ }^{201} \mathrm{Tl}\right)$ and dual-radionuclide $\left({ }^{201} \mathrm{Tl}+{ }^{99 \mathrm{~m}} \mathrm{Tc}\right)$ SPECT images of a $1-\mathrm{cm}$ insert placed in the anterior myocardial wall of the anthropomorphic torso phantom show blurring and diminishing of the defect on the 


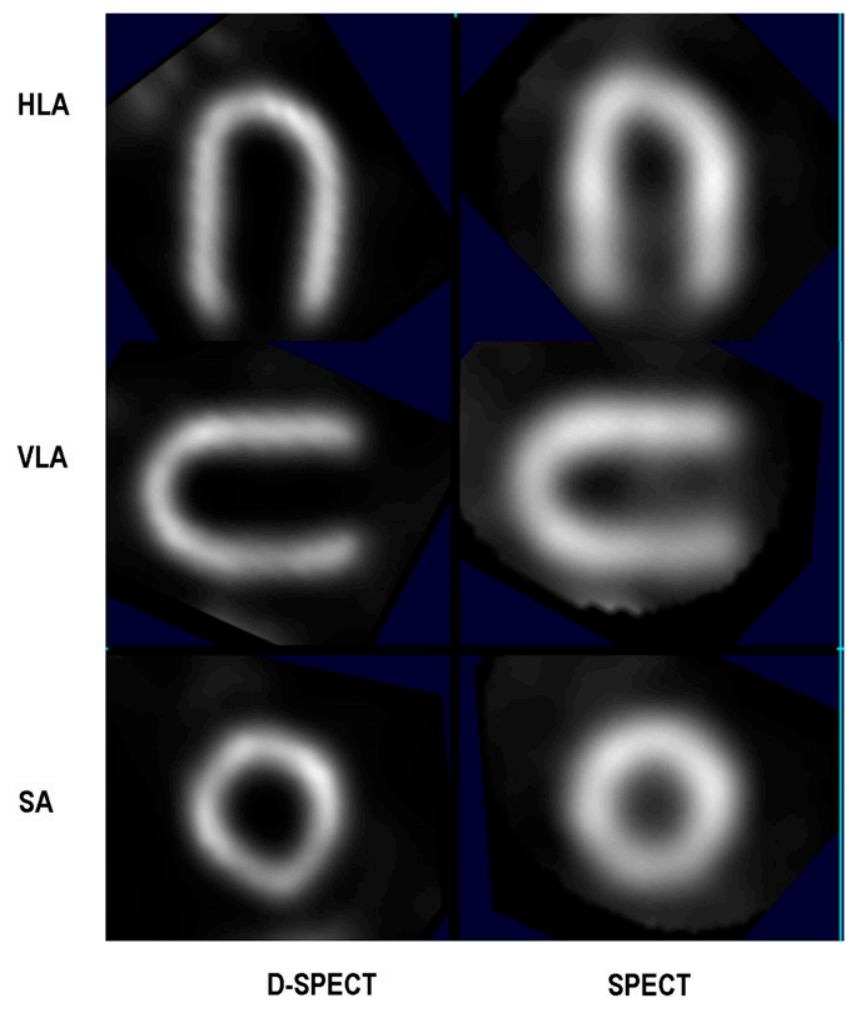

FIGURE 4. Images of anthropomorphic torso phantom scanned on both D-SPECT and SPECT. Only myocardial portion of image is shown. Horizontal long-axis (HLA), vertical long-axis (VLA), and short-axis (SA) cuts are shown. For additional examples, see Supplemental Figure 6.

conventional-SPECT images when obtained under the influence of the ${ }^{99 \mathrm{~m}} \mathrm{Tc}$ cross-talk. This image degradation is almost not perceivable on the D-SPECT images, demonstrating preserved lesion detection with simultaneous dualradionuclide SPECT (Supplemental Fig. 7).

\section{Patient Studies}

Given the highly encouraging results with line sources and the physical torso phantom, we next proceeded to patient imaging studies. Overall patient image quality was rated good and higher in 17 (94\%) cases for D-SPECT and 16 (89\%) cases for SPECT; D-SPECT images showed qualitatively better myocardial edge definition than did SPECT images in all cases, as assessed by 4 masked nuclear medicine physicians. D-SPECT SSS and SRS correlated linearly with SPECT respective scores $(r=0.83, P<0.0001$, for SSS, and $r=0.76, P<0.001$, for SRS, with a slope of 0.98$)$. Of the 18 studies, $14(78 \%)$ were rated as definitely normal or abnormal for both SPECT and D-SPECT. The myocardial counting rate was significantly higher in D-SPECT than in SPECT $(384,000 / \mathrm{min} \pm 134,000 / \mathrm{min}$ vs. $47,000 / \mathrm{min} \pm 14,000 /$ min, respectively $[P<0.0001]$ for stress and $962,000 / \mathrm{min} \pm$ $426,000 / \mathrm{min}$ vs. $136,000 / \mathrm{min} \pm 37,000 / \mathrm{min}$, respectively $[P<0.001]$ for rest). Three independent nuclear medicine physicians with no affiliations to Spectrum Dynamics also independently read the studies, and image quality was rated good and higher in $98 \%$ cases for D-SPECT and 92\% cases for SPECT; again, D-SPECT images showed qualitatively better myocardial edge definition than did SPECT images in all cases. End-diastolic and end-systolic images from the gated SPECT data in 2 patients are shown in Supplemental Figure 8. Superior edge definition with D-SPECT is again evident. Table 1 shows the corresponding ejection fractions and end-diastolic and end-systolic volumes estimated from the images. In patient 1 (Fig. 5), normal rest/stress myocardial perfusion was observed, with no significant defects noted. In patient 2 (Supplemental Fig. 9A), who had a history of myocardial infarction, there was a fixed perfusion defect in the anteroseptal and apical regions with no stress-induced defects. In patient 3, who had atypical angina (Supplemental Fig. 9B), there was a reversible stress-induced defect in the inferior wall.

\section{DISCUSSION}

The D-SPECT camera provides a novel photon-collection method and scanning geometry that includes position and orientation tracking of multiple collimated detector columns moving independently. The D-SPECT has been developed to improve the built-in conflict between spatial resolution and sensitivity inherent in conventional-SPECT camera systems. Tungsten collimators, with larger holes and a shorter length than the standard lead parallel-hole collimators used for SPECT, result in an acceptance angle approximately 8 times that of the standard lead, parallel-hole collimators, thus improving system sensitivity. The potential loss of spatial resolution because of wider-bore, shorter collimators is more than compensated for by incorporating a model of the collimator response in the reconstruction algorithm. By tracking the positions and orientations of the detectors at all times relative to the scanned organ, the system can record the changes in counts as the detectors are moved through small angular increments, allowing collimation with a greater acceptance angle while increasing spatial resolution.

The line source, torso phantom, and clinical studies of this report document an order of magnitude increase in sensitivity, with improvement in reconstructed spatial resolution, compared with the conventional-SPECT technol-

\begin{tabular}{|c|c|c|c|c|c|c|c|}
\hline \multirow[b]{2}{*}{ Patient } & \multirow[b]{2}{*}{ Modality } & \multicolumn{3}{|c|}{ Stress } & \multicolumn{3}{|c|}{ Rest } \\
\hline & & $\overline{E F}$ & ESV & EDV & $\overline{E F}$ & ESV & EDV \\
\hline \multirow[t]{2}{*}{ A } & D-SPECT & 54 & 40 & 87 & 57 & 34 & 79 \\
\hline & SPECT & 52 & 34 & 71 & 60 & 28 & 71 \\
\hline \multirow[t]{2}{*}{ B } & D-SPECT & 27 & 140 & 193 & 28 & 141 & 198 \\
\hline & SPECT & 29 & 137 & 195 & 29 & 128 & 182 \\
\hline
\end{tabular}




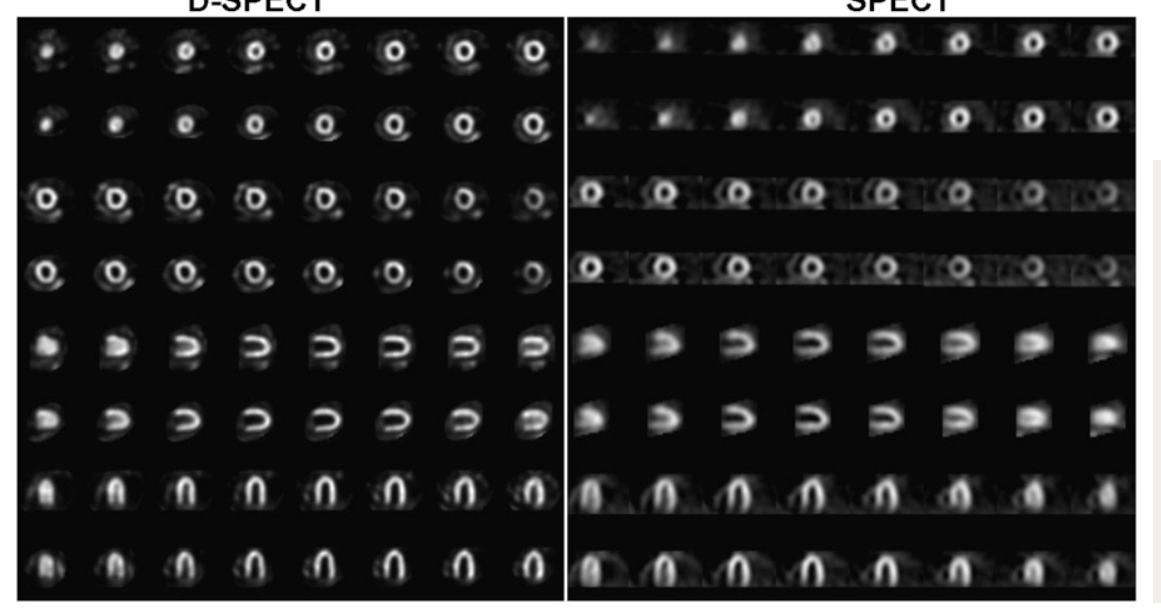

FIGURE 5. Comparative images from a patient. From top to bottom, D-SPECT and SPECT tomographic slices in mid short axis (2 levels), mid vertical long axis, and mid horizontal long axis are shown. Top row of each set are stress images, and bottom row of each set are rest images. Patient had no known cardiac history and had smoking as risk factor, and no perfusion defects are noted. For 2 more patient examples, see Supplemental Figure 9.

ogy that is currently used in clinical practice. The marked increase in sensitivity results in much shorter acquisition times than those obtained with SPECT. The sensitivity and short detector rotation time of the D-SPECT camera provide the potential for serial brief tomographic acquisitions (as short as $10 \mathrm{~s}$ ) during a single study, that is, dynamic SPECT. In terms of absolute counting rates, the UFOV phantom study revealed a sensitivity of 0.127 counts/Bq/min. These observed absolute counting rates for the D-SPECT camera are greater than 10 times those generally acknowledged for a dual-detector system equipped with LEHR collimators (6). In addition to documenting the achievement of high-quality perfusion images in $2 \mathrm{~min}$, the gated SPECT example of patients in this study shows that this brief acquisition can also provide high-quality images of ventricular function. The sensitivity advantages of the D-SPECT camera will be volume-dependent, and the gains reported here from heart imaging may not necessarily hold as other organs are imaged. Sensitivity can also be increased by using special collimators on a conventional dual-head SPECT system, and future studies should look into such comparisons. Even with such special collimators, the D-SPECT smart rotation of detectors makes it unique.

The superior spatial resolution of the D-SPECT system noted in the various phantom studies was confirmed in the patient studies, with better edge definition of the myocardium noted in all studies. Estimation of reconstructed spatial resolution when using iterative reconstruction is problematic, however. Because of the nonlinear properties of iterative reconstruction, spatial resolution is variable and object-specific. Nevertheless, it is useful to estimate spatial resolution, to demonstrate the system properties relative to conventional SPECT. The use of a perturbation approach as described estimates resolution at image locations of clinical importance, illustrating that the resolution of the D-SPECT camera is better than that obtained in current clinical practice and even approaches the best performance achievable when modeling resolution with a high-resolution collimator. The use of task-dependent metrics based on contrast versus noise and observer studies are alternatives that will need to be explored in future work. Future comparisons of the resolutions of the SPECT and D-SPECT systems should also explore reconstructed resolution compared at equal noise levels, and noise levels should be compared at equal recovered resolutions.

Image quality is a key component in accurate image interpretation and influences confidence in the interpretation. The superior image quality associated with the D-SPECT system should thus translate into more accurate and fewer ambiguous interpretations than are observed with SPECT. Potentially, the D-SPECT molecular imaging technology can be integrated with a wide range of existing modalities including CT, MRI, and ultrasound systems, providing a fused-imaging modality. Coupling of the new camera device to a CT system, for instance, will provide the capability for registered acquisition of attenuation maps for high-quality attenuation corrections to be performed on the emission scans, but the patient positional differences (supine vs. sitting) will have to be addressed. The improved spatial resolution and sensitivity of the D-SPECT system should make it possible to take better advantage of the complementary information provided by the CT scanner. In turn, image quality of the attenuation-corrected emission scans will improve. Among other advantages of the D-SPECT system, the marked increase in sensitivity and spatial resolution could potentially enable the use of low-dose radiopharmaceuticals, dynamic imaging with absolute measurements of tissue perfusion, and parametric representation of underlying biochemistry. Additionally, the increase in sensitivity and spectral resolution could allow the use of multiple radiopharmaceutical cocktails, creating vast opportunities for multidimensional mapping of disease processes.

The D-SPECT system uses solid-state CZT detectors, permitting a unique geometry of detectors that would not be feasible with the conventional thick crystals and large photomultiplier tubes that characterize the Anger camera. Tungsten collimators provide better count sensitivity than those made from lead. The superior stopping power of 
tungsten, compared with lead collimation, allows thinner collimator septa, thus increasing the useful detector area.

Beyond the benefits of improved sensitivity and spatial resolution, the D-SPECT system geometry allows the system to be significantly miniaturized and ergonomically optimized for both the patient and the user. Newer clinical reconstruction algorithms such as Astonish (Philips Inc.), Flash 3D (Siemens Inc.), and Evolution (GE Healthcare) may also improve spatial resolution, and future studies should directly compare these methods with the D-SPECT system. Other commercial systems that will also eventually need to be compared with D-SPECT include, but are not limited to, the CardiArc (CardiArc Inc.), IQ SPECT (Siemens Inc.), and C-SPECT/CT (formerly referred to as MarC-SPECT) systems.

The expected benefits related to the increased sensitivity of the novel D-SPECT technology in cardiac imaging include dynamic SPECT acquisitions that will allow kinetic perfusion tracer modeling and measurements of absolute myocardial perfusion and coronary flow reserve, potential reduction of radiation exposure to the patient by the lowering of the radiopharmaceutical dose, and improved patient comfort because of rapid examinations. Additionally, the potential exists for simultaneous rest/stress imaging using dualradionuclide myocardial perfusion SPECT, because of the improved energy discrimination of the CZT detectors but also because of the potential trade-off of the decreased imaging time for a reduction in activity (with consequently reduced spillover [cross-talk] between the radionuclides). Routine simultaneous dual-radionuclide myocardial perfusion SPECT would further reduce by a factor of 2 the time associated with a rest/stress myocardial perfusion SPECT study. The D-SPECT technology, when combined with precise and unique radiopharmaceutical protocols, could make molecular imaging more disease-specific through a tissuesignature database. This technology could potentially significantly change the conventional medical diagnostic process. One of the key limitations of SPECT has been the inability to perform dynamic imaging with a high temporal resolution (multiple images to allow the monitoring of tracer kinetics). The D-SPECT technology — with further validation — should allow dynamic sampling, and therefore, time-activity curves could be obtained.

\section{CONCLUSION}

We presented the initial performance characteristics of the D-SPECT technology for cardiac perfusion imaging. These initial performance characteristics of the D-SPECT camera, including markedly improved sensitivity with spatial resolution recovery and higher patient throughput, offer great promise for clinical dynamic SPECT protocols. The D-SPECT camera should have important implications for applications in nuclear cardiology and eventually molecular imaging.

\section{ACKNOWLEDGMENTS}

We thank Dalia Dickman, Benny Rousso, Eli Dichterman, Omer Ziv, Haim Melman, Yoel Zilberstein, Nathaniel Roth, Ran Ravhon, and Samy Bross at Spectrum Dynamics for all their hard efforts in helping to develop, improve, and evaluate this novel technology. We acknowledge the independent rereads of the patient images by Drs. Sean Hayes, Arik Wolak, and Ariel Gutstein in the Imaging Department at Cedars Sinai Medical Center in Los Angeles, California. We thank Todd Peterson, for helpful editorial comments, and Xingping Kang, for assistance in preparation of this manuscript. This study was funded in part by the U.K. Department of Health's NIHR Biomedical Research Centers funding scheme. Drs. Daniel Berman and Shlomo Ben Haim own shares in Spectrum Dynamics. Drs. Daniel Berman, Martin Sandler, Jack Ziffer, and Sanjiv Gambhir are members of the Medical Advisory Board and consultants for Spectrum-Dynamics. Drs. Sanjiv Gambhir, Jim Patton, and Brian Hutton are members of the Spectrum-Dynamics Technical Advisory Board.

\section{REFERENCES}

1. Berman DS, Hachamovitch R, Shaw LJ, Germano G, Hayes S. Nuclear cardiology. In: Fuster V, Alexander RW, O'Rourke RA, eds. Hurst's The Heart. New York, NY: McGraw-Hill; 2004:563-598.

2. Klocke FJ, Baird MG, Lorell BH, et al. ACC/AHA/ASNC guidelines for the clinical use of cardiac radionuclide imaging: executive summary-a report of the American College of Cardiology/American Heart Association Task Force on Practice Guidelines (ACC/AHA/ASNC Committee to Revise the 1995 Guidelines for the Clinical Use of Cardiac Radionuclide Imaging). Circulation. 2003;42:1318-1333.

3. Anger HO. Scintillation camera with multichannel collimators. J Nucl Med. 1964;5:515-531.

4. Babla H, Bai C, Conwell S, et al. A triple-head solid state camera for cardiac single photon emission tomography (SPECT). Proc SPIE. 2006;6319: 63190M.1-63190M.5.

5. Darambara DG. Solid state detectors in nuclear medicine. $Q \mathrm{~J} \mathrm{Nucl} \mathrm{Med}$. 2002;46:3-7.

6. Wagenaar DJ. CdTe and CdZnTe semiconductor detectors for nuclear medicine imaging. In: Wernick MS, Aarsvold JN, eds. Emission Tomography: The Fundamentals of PET and SPECT. Amsterdam, The Netherlands: Elsevier Academic Press; 2004:269-291.

7. Kim H, Furenlid LR, Crawford MJ, et al. SemiSPECT: a small-animal singlephoton emission computed tomography (SPECT) imager based on eight cadmium zinc telluride (CZT) detector arrays. Med Phys. 2006;33:465-474.

8. Brzymialkiewicz CN, Tornai MP, McKinley RL, et al. Evaluation of fully 3-D emission mammotomography with a compact cadmium zinc telluride detector. IEEE Trans Med Imaging. 2005;24:868-877.

9. Mueller B, O'Connor MK, Blevis I, et al. Evaluation of a small cadmium zinc telluride detector for scintimammography. J Nucl Med. 2003;44:602-609.

10. Tsui BMW, Gunter DL, Beck RN, et al. Physics of collimator design. In: Sandler MP, Coleman RE, Patton JA, et al., eds. Diagnostic Nuclear Medicine. Vol 1. 3rd ed. Baltimore, MD: Williams and Wilkins; 1996:67-79.

11. Cherry SR, Sorenson JA, Phelps ME. Physics in Nuclear Medicine. 3rd ed. Oxford, England: W.B. Saunders Co.; 2003:227-251.

12. Gunter DL. Collimator design for nuclear medicine. In: Wernick MS, Aarsvold $\mathrm{JN}$, eds. Emission Tomography: The Fundamentals of PET and SPECT. Amsterdam, The Netherlands: Elsevier Academic Press; 2004:153-168.

13. Formiconi AR. Geometrical response of multihole collimators. Phys Med Biol. 1998;43:3359-3379.

14. Beekman FJ, Vastenhouw B. Design and simulation of a high-resolution stationary SPECT system for small animals. Phys Med Biol. 2004;49:4579-4592.

15. Borges-Neto S, Pagnanelli RA, Shaw LK, et al. Clinical results of a novel wide beam reconstruction method for shortening scan time of Tc-99m cardiac SPECT perfusion studies. J Nucl Cardiol. 2007;14:555-565.

16. King MA, Glick SJ, Pretorius PH, et al. Attenuation, scatter, and spatial resolution compensation in SPECT. In: Wernick MS, Aarsvold JN, eds. Emission 
Tomography: The Fundamentals of PET and SPECT. Amsterdam, The Netherlands: Elsevier Academic Press; 2004:74-89.

17. Frey EC, Tsui BMW. Collimator detector response compensation in SPECT. In: Ziadi H, ed. Quantitative Analysis in Nuclear Medicine Imaging. New York, NY: Springer; 2006:141-166.

18. Tsui BMW, Hu HB, Gilland DR, Gullberg GT. Implementation of simultaneous attenuation and detector response correction in SPECT. IEEE Trans Nucl Sci. 1988;35:778-783.

19. Hutton BF, Lau YH. Application of distance-dependent resolution compensation and post-reconstruction filtering for myocardial SPECT. Phys Med Biol. 1998;43:1679-1693.
20. Hudson HM, Larkin RS. Accelerated image reconstruction using ordered subsets of projection data. IEEE Trans Med Imaging. 1994;13:601-609.

21. Segars WP, Lalush DS, Tsui BMW. A realistic spline-based dynamic heart phantom. IEEE Trans Nucl Sci. 1999;46:503-506.

22. Germano G, Kiat H, Kavanagh PB, et al. Automatic quantification of ejection fraction from gated myocardial perfusion SPECT. J Nucl Med. 1995;36:21382147.

23. Stayman JW, Fessler JA. Fast methods for approximation of resolution and covariance for SPECT. In: Conference Record IEEE Nuclear Science Symposium and Medical Imaging Conference: 10-16 November 2002, Norfolk, Virginia, USA. Vol 2. Piscataway, NJ: IEEE; 2003:786-788. 